\title{
VeSMEL: A Novel Approach for Distance Management of E-Learning Platforms.
}

\author{
doi:10.3991/ijet.v6iS2.1650 \\ B. Batchakui, T.N. Djotio and C. Tangha \\ University of Yaoundé, Yaoundé, Cameroon
}

\begin{abstract}
This article presents a novel approach for distance management for e-learning platforms based on VeSMp protocol. VeSMEL results from the integration of VeSMp to xMoodle 2.0 platform that has been developed. It offers a compromise between the mobility of actors and the availability of xMoodle 2.0 content in zones where internet is inaccessible. The GSM system is used like support for communication allowing the actors to reach servers from their cell phone.
\end{abstract}

Index Terms-E-learning; GSM; Remote monitoring and teleconsultation; VeSMp; xMoodle2.0.

\section{INTRODUCTION}

Nowadays, the majority of e-learning platforms are based on Internet technology (distant access through IP protocol). The use of such platforms requires either the presence on the sites where applications are installed or the availability of Internet connection. These constraints do not facilitate the mobility of their actors (learners, authors, and teachers). For example, it will not be possible in a zone without connection to interact with the platform to get information about the availability of the contents or to even disconnect a user on the basis of nonpayment. Thus, the developing countries where the digital fracture remains very high do not profit from the teaching assets which these platforms offer.

Furthermore, the GSM network (Global System for Mobile communication) just like electrical communication has a higher density than the Internet network. In Cameroon for example, the statistics published in 2007 by the NTIC regulatory board [9], reveal that the coverage of the mobile network is about $60 \%$ whereas that of the Internet is about $0,13 \%$. In addition, the mobile phone is accessible to a large majority of the population.

Djotio, within the framework of his $\mathrm{PhD}$ thesis proposes a protocol based on SMS named VeSMp [3], which makes it possible via the mobile phone to manage a remote network.

In addition, we developed a distribution of Moodle, named xMoodle 2.0 [6]. The later is the result of integration with Moodle of a module that is able to transform the most current none structured contents (course with the format .doc, pdf, and HTML), to structured contents with the format xml. As derived from Moodle, xMoodle2.0 is based on Internet.

How can the management of xMoodle2.0 be extended to zones which do not have Internet? In other words how can the xMoodle 2.0 services be offered via a GSM network? The proposed approach to the solution to this question, the subject of this research rests on the protocol
VeSMp (Very Short Message Protocol), which is a particular protocol based on GSM technology, it allows the administrator of a system/network to take control of a remote network. VeSMEL is an adaptation of VeSMp in a particular context of e-learning where the services to be reached are provided by a LMS (Learning Management System). This is coupled to a convivial interface for automatic editing of commands on mobile phones.

This article is structured as follows: initially the problem of distant management of the platforms is posed, then our contribution based on the design and implementation of VeSMEL is presented, followed by the results obtained. Finally a discussion of the results and the conclusion are presented.

\section{MANAGEMENT OF AND E-LEARNing PlatForm}

The management of an e-learning platform is viewed essentially from three angles (administration, teaching, and learning):

- Administration includes account management functions (registration of users in the platform, connection and disconnection of users, etc), service configuration, insertion and removal of contents in the platform, etc.

- Teaching has to do with creation and provision of the contents for the platform

- Learning is made up of the training tools such as consulting and reading of the contents, the downloading and remote uploading of contents of assignments, etc.

Regardless of any of these angles there are two standards types of management:

- Local management: in this case one has a server in a room which shelters the platform and to which computers of a local area network are connected. The actors connect to it. This kind of management does not offer the possibility of mobility to its users, and moreover, it is limited to the number of work stations of the local area network for this purpose.

- Distant Management: to permit access to the contents to a large audience, it is necessary to exceed the framework of the local area network; we need a wide area network. In developing countries, that supposes very heavy investments. Consequently, it is necessary to go towards less expensive infrastructures which support mobility and the access to a large audience; Internet is the solution. Moodle and consequently xMoodle 2.0 as we announced above are based on Internet. Such platforms on an Internet network would cover a very large public and ensure the 
mobility of its users. But in this context, the digital divide is very high as mentioned above. The problem is how to allow the greater part of the learners to profit from the assets of a platform like xMoodle 2.0.

The mobile network is highly densed. It is accessible almost everywhere. Majority of the people have a mobile phone. Why can this infrastructure not be used to offer broad access and mobility to the platform xMoodle 2.0? Many projects for the mobility of the Moodle platform can be identified [11]; we can mention MOMO (Mobile Moodle) which has been abandoned, OrangeWhitepda which had serious display problems and those of ergonomics, and MLE-Moodle (Mobile Learning Engine) which is a modification of MOMO. Majority of these projects are very recent (around 2009), generally complete and based on the Internet.

The GSM Network is a good alternative which is based on a particular protocol called VeSMp [1]. Indeed, it is a protocol based on SMS technology of GSM. It allows the administrator of a system/network to take control of a remote network. The SMS are quite simply transformed into system commands and sent to computers that must be monitored. Indeed, it enables SMS messages to act as PDUs (Protocol Data Units) in which the system commands are conveyed in the same manner as the SDUs (Service Dated Units) [1]. Thus, by this protocol, the cell phone becomes a complementary tool for administration of a network/system.

The administrator gives instructions to carry out tasks starting from a grammar developed around VeSMp. This grammar is currently limited to the network administration tasks. This work offers an extension of VeSMp grammar to the E-learning platforms and a possibility for ordinary users to use this protocol to access training contents using the mobile phone independent of their geographic location by a simple emission of commands.

VeSMp grammar is limited to the control of administration network and the administration is done through the standard data entry screen of the mobile phone. We propose to extend VeSMp to the management of the platforms; this requires an extra effort because the network commands administrations are often well-known and are not heavy primitives. On the other hand the management of a platform requires the combination of several commands. Furthermore, the issuing of commands is not as convenient as in system administration; consequently a convivial interface access to the services of the platform must be provided. The interfaces proposed are buttons which encapsulate the commands to be sent to the server for interpretation. The lines bellow present the analysis and design of VeSMEL.

\section{ANAlysis AND Design OF VeSMEL}

\section{A. Logical organisation}

VeSMEL is a unit made up of three modules:

- A customer module, include software tools deployed in mobile phones. These are the tools which command xMoodle 2.0 remotely. The application recovers the requests of the user through interfaces, then generates VeSM. VeSM generated are sent to the connection module which has the responsability to decode them and send them to the server module for xMoodle 2.0 platform.

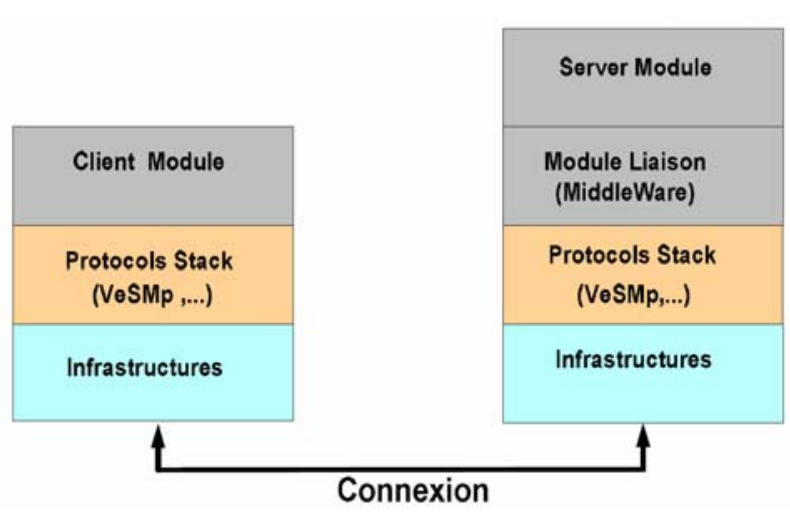

Figure 1. System logical architecture

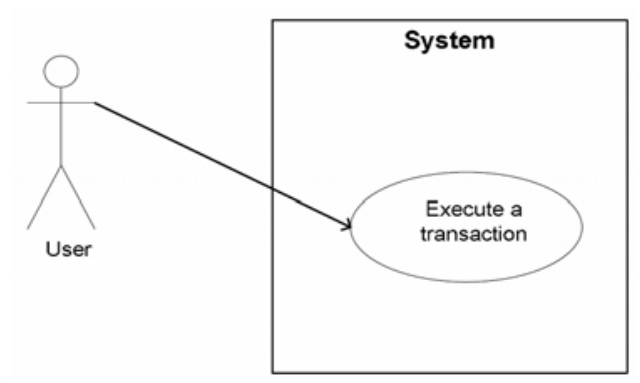

Figure 2. Use case diagram

- A connection module, which includes the software tools developed to receive the message of the client phone and transfer it to the computer in which xMoodle 2.0 is installed after intermediate processing. It plays the role of middleware between the client module and server module.

- And a server module, which is used for administration. It contains software tools which recover the parameters coming from the mobile phone, connects itself to xMoodle 2.0 to provide the functions of administration.

Infrastructures, layers and stack of protocols are admitted as hypothesis; consequently they are not our preoccupation. Figure 1 presents the logical architecture of VeSMEL.

\section{B. Use case diagram}

VeSMEL allows any person that uses xMoodle 2.0 platform, to reach information of the latter by a mobile phone. The user thus executes a transaction starting from a client module installed on his mobile phone. A transaction is either a transaction of access to contents or administration transaction. The use case diagram is presented in figure. 2.

The execution of a transaction requires the exchange between two modules of the system: The client module of the mobile phone called "Client" and the server module of the xMoodle 2.0 server called "Server".

The transactions sent are based on the grammar presented in the design part below.

\section{Grammar associated with VeSMEL}

\section{1) Vocabulary} ries: 
- Actions related to the management of the lessons, consisting of the following transactions: add a course (Ajcc), consult the available courses (Lcc), consult the teachers who intervene in a course (Ecc), consult the schedules of a course (Hcc), consult learners that registered for a course (LAcc), consult work submitted by learners (Dcc), consult the frequently asked questions relative to a given course (Qcc).

- Actions that relate to the management of users consists of the following transactions: the list of learners registered (LiUser), the list of learners connected (LcUser), the list of regular learners (LrUser), disconnect a user (DecUser), connect a user who was disconnected (ConUser).

- And actions relating to startup of the platform consist of the following transactions: start the platform (UpSvr), and stop platform (DwnSvr).

From these types of actions, a complementary vocabulary called "Ve" can be built, it comes to enrich the vocabulary "V" of VeSMp [3]:

$\mathrm{Ve}=\{$ Ajcc, Lcc, Ecc, Hcc, Dcc, Qcc, LiUser, LcUser, LrUser, DecUser, ConUser, UpSvr, DwnSvr $\}$ in addition to \#\#, \#, @ which are special characters of $\mathrm{V}$, used to form commands. In particular @ is used to specify an address of machine.

We specify that this vocabulary was adopted for the xMoodle 2.0 platform. For any other platform, we planned a convention on which it is necessary to be based. The elements of the vocabulary that represent commands UpSvr and DwnSvr are adopted whatever the platform of e-learning, it is the same for the special characters \#\#, \#, and @. The other commands follow the semantics below: CmdPlateforme $=$ [notation] [Suffix]

Where:

- CmdPlateforme is the command,

- and notation is the short chain (with not more than three characters) related to the transaction to be carried out.

In the case of the platform xMoodle 2.0, the notation belongs to $\mathrm{N}=\{\mathrm{Aj}, \mathrm{L}, \mathrm{Li}, \mathrm{LLC}, \mathrm{Lr}, \mathrm{DEC}, \mathrm{H}, \mathrm{E}, \mathrm{Q}$, Con, Up, Dwn $\}$ and Suffixe belongs to $S=\{D C$, User $\}$.

2) Syntax

The syntax of use or exploitation is the following one: \#\#SeqNum\#\#Plateforme\#CmdPlateforme\# [Parameter] [@Machine].

- Platform represents the name of the platform.

- CmdPlateforme represents the command to be carried out at the level of the platform.

- Parameter is a succession of character strings preceded by \#, it is the list of the parameters associated with the command.

- Machine represents the address of the machine on which the platform is installed.

3) Associated Grammar

VeSMEL comes to enrich VeSMp grammar to enable it to support the platforms of e-learning. Associated grammar arises as follows:

VeSM ----- > \#\#ListeCommande (see [1]).

ListeCommande ----> CmdConnexion | CmdDeconnexion | ListeCmdPilotage | CmdPlateforme.
We are thus interested in the e-learning aspect in "CmdPlateforme".

CmdPlateforme ----->SeqNum\#\#Plateforme\# CmdExecutable.

CmdExecutable------>CmdVe\#Parametre (CmdVe is an order resulting from the Ve vocabulary presented in 1 above).

Parameter ---->\#chaine_1\#... \#chaine_i\#... \#chaine_n\# (Series of chains surrounded by \#).

\section{Example of a command:}

\#\#212\#\#xMoodle2.0\#LiUser\#Nomuser\#PrenomUser\#D ateI-nscrit\#@192.1.168.5

This command returns the list of the registered students: 212 represents the number of sequence and xMoodle 2.0 represents the platform (it is with the 192.1.168.5 address). The resulting list will be organized by name, first name and date of inscription.

\section{Design model}

The execution of a transaction is carried out during the exchange between the following objects:

- FrmClient, is the form on which the parameters of the requests are recovered on the mobile phone side (client station).

- Commande_Manager, is used to construct commands.

- SmsManager, manages, send and receives messages.

- CoursFrm, contains display operations of contents.

- UserFrm, contains the operations of management of xMoodle2.0 users.

Figure 3 presents a simple form of VeSMEL system sequence diagram. We put forward only the basic objects. In this article we make emphasis to client stations (mobile phone).

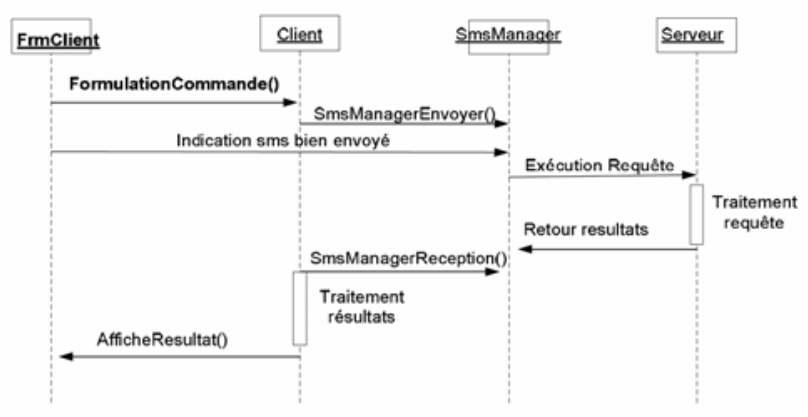

Figure 3. System sequence Diagram

The classes below are added to the classes resulting from the objects presented above:

- BaseFrm, basic class of the forms (FrmClient, CoursFrm and UserFrm are his heirs).

- And ActsFrm, for the management of other actions.

The above interaction diagram results from the class diagram of figure 4 . 


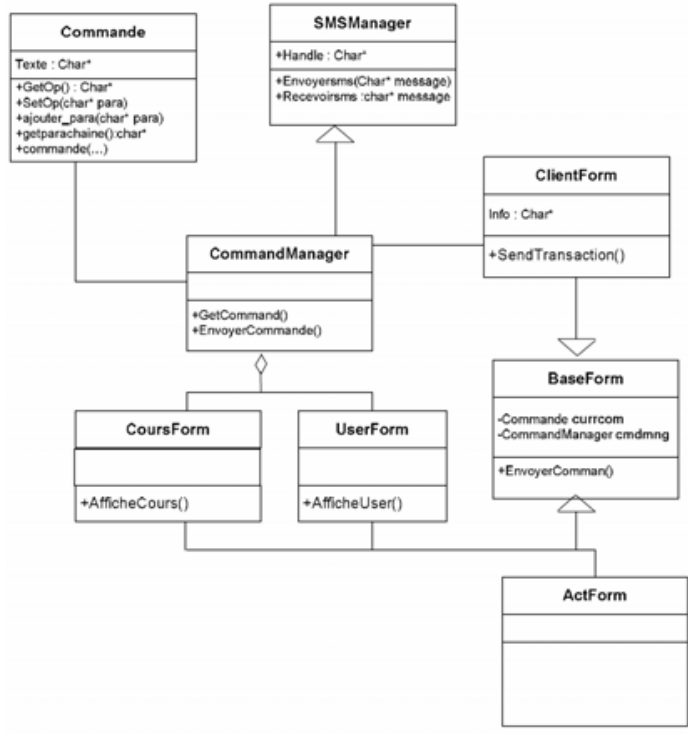

Figure 4. Class Diagram

\section{IMPLEMENTATION}

The implementation is based on the J2ME technology. J2ME (Java 2 Micro Edition) is a framework dedicated to the development of java applications which are running in mobile phones. J2ME resulted from the desire of SUN to define the Java specifications for target devices having low capacities. These specifications are organized around a set of configurations and profiles:

- Configurations define the basic characteristics of an environment for execution of a certain type of machine having several similar characteristics and resources. They include a virtual machine and all of basic API. The currently definite configurations are the CLDC (Connected Limited Device Configuration) and the CDC (Connected Device Configuration).

- Profiles are composed of a set of particular API specific to a type of machine in terms of functionality. They allow the use of precise functionalities and must be associated with a configuration. It ensures a certain modularity of J2ME platform.

The development of J2ME applications starts with the definition of the capacity of the target devices (configura- tion), followed by the choice of the profile to make it possible for the system to load the API necessary for the execution of the application. The choice of J2ME is justified by the fact that today; the greater majority of mobile environments carry out applications J2ME comfortably.

\section{RESUlts}

In this section relating to the results, we present some user interfaces obtained. At the functional level, the launching of a transaction proceeds as follows:

- A click on the link of the transaction generates a message in the direction of the Java server installed on the xMoodle 2.0 station.

- The server recovers the arguments encapsulated in the message and sends it to HTTP client.

- HTTP client carries out a request towards xMoodle 2.0, recovers the answer, analyses and extracts important information.

- The important information is returned to the client Module.

- And client module displays the information received.

Figure 5, presents the connection interface of the mobile client to the platform. The login and the password are encapsulated in the form of commands sent as specified above.

Figure 6, presents the mobile client menu. The implemented sub-modules are:

- The accounts management "Compte" which allows adding, modifying, and suppressing of an account.

- The courses management "Course", which makes it possible to consult the courses available, to know the teachers or persons in charge of a given course, learners registered for a given course.

- The control "controles", which enables or disables an account. For a given course, it enables or disables participants.

- The contents access “CoursCo", which makes it possible to consult the list of concepts of a course and to display a given concept.

Figure 7, presents an example of a management screen for courses with the list of teachers of a course entitled Object Oriented Programming with course code (Code EU) POOII.

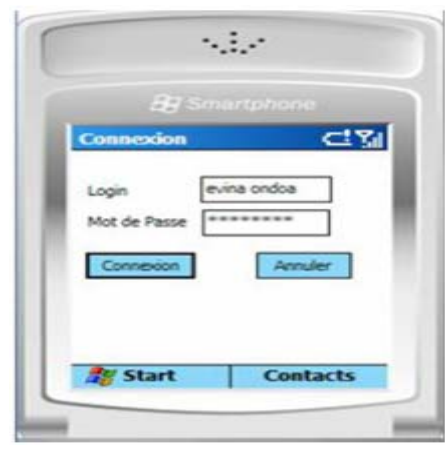

Figure 5. Connection interface of the mobile client

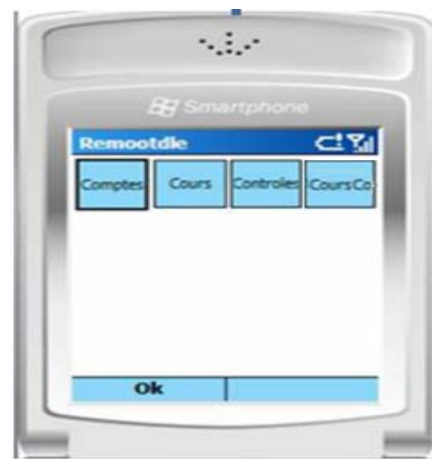

Figure 6. Menu client Mobile phone.

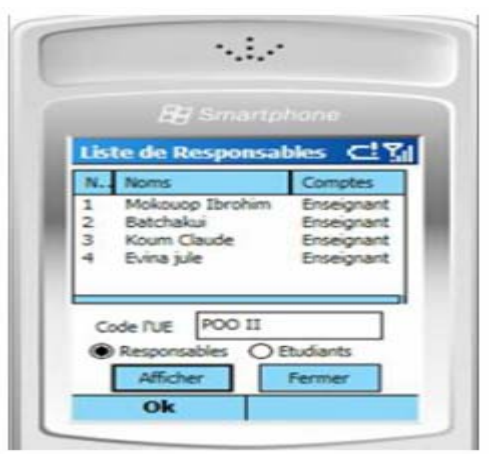

Figure 7. List of the persons in charge of a given course 


\section{DISCUSSIONS AND CONCLUSION}

VeSMEL, a system which has the functionalities of management of accounts and contents (creation of users, connection and disconnection of a user, information on the contents, etc) has been presented. It constitutes an interesting compromise between the mobility of the actors of a platform of FOAD (Formation Ouvert A Distance) [4] and the availability of information that the latter needs to be more efficient.

Based on the VeSMp protocol, it extends the use of the platforms of FOAD to many users of developing countries where the digital coverage is very weak, and even those in developed countries. Within the framework of this work, we put an accent on the xMoodle 2.0 platform, a distribution of Moodle developed by our team [6]. The adopted approach is certainly generic at the level of the grammar design and requires more studies for any other platform different from xMoodle 2.0 (development environment, basic standard of data supported, structure of the handled tables, etc). The security issues are those posed by VeSMp and it's taken into account in the work of [3].

Java using J2ME library was adopted for the development of the client module, because it covers a broad range of mobile phones. But to be remaining more open, the development of a $\mathrm{C}++$ customer is necessary (it is currently being studied).

VeSMEL will be integrated like a service of the GLS (Grid Learning Services) of GELSOTC under development at the ENSP of Yaounde-Cameroun [2, 8], which will extend its use to the platforms of the heterogeneous and distributed environments.

The grammar "Ve" currently covers some functions of administration and use of the platform xMoodle 2.0. It continues to be studied. It is required that it should be generic and that it should cover all the transactions used in the management of the platform xMoodle2.0.

\section{ACKNOWLEDGMENTS}

We are grateful to numerous colleagues and friends for discussions on the topics covered in these pages, in particular Professor Roger NKAMBOU of the University of Montreal in Canada, Professor Jean Claude Derniame of the IPL-France, and Mr. Edwin MBINKAR Lecturer at the department of Electrical Engineering ENSP-YaoundéCameroon.

\section{REFERENCES}

[1] Djotio, T. N., Tangha, C., and Kouamou G. E.,. VeSMp (Very Short Message): An SMS-Based Protocol for Process Monitoring and System Remote Administration. Proceedings of International Joint Conference on e-Business and Telecommunication- ICETE 2007 (IBSN 978-989-15-9), pp 25-32, june 2007.

[2] Batchakui, B., Tangha, C., Nkambou, R., Djotio T. N.,. Apprenticeship Environment and Co-operation Based on the Grid and Web 2.0 Designed for Training Communities with Commun Interest. ICCR'09 pp 311-328, 2009.

[3] Djotio, T.N., 2008, Contribution à l'Administration et à la Sécurité Réseau: Very Short Message Protocol (VeSMp) et Modèle d’Ontologie Centré sur la Gestion d’Intrusions réseaux (MOCGIR), Thèse de Doctorat PhD, Ecole Nationale Supérieure Polytechnique, Université de Yaoundé 1, 2008.

[4] Hahemat Mahamat, Modèle de classification des apprenants en FOAD pour une assistance adaptée, Mémoire de DEA, Ecole Polytechnique Yaoundé, 2005.
[5] Kim Topley, J2ME in a Nutshell. O'Reilly, Edition March, 2002 (ISBN: 0-596-00253-X, 478 pages).

[6] Batchakui, B., Tangha, C., Nkambou, R., Djotio T. N.,. xMoodle2.0 : Une perspective pour la diminution de la charge cognitive de l'apprenant dans la plateforme Moodle. Proceeding of the 10th African Conference on research in computer Science and Applied Mathematics. CARI'10, october 2010 Yammoussoukro, pp 477.

[7] R. Yatchou, "Réduction de la distance Transactionnelle et amélioration du contexte social en télé-enseignement sur Internet", Thèse de Doctorat $\mathrm{PhD}$, Ecole Nationale Supérieure Polytechnique, Université de Yaoundé 1, 2001.

[8] B. Batchakui, C. Tangha, R. Nkambou, G. Kouamou, «Environnement de collaboration basé sur le Grid Learning Services (GLS) pour les communautés de formation à centre d'intérêt commun» quatrième conférence -I2LOR 2007, 7 novembre 2007 à Montréal thème: «Intelligent, Interactivelearning Object Repositories ».

[9] ANTIC. Données statistiques au Cameroun de la téléphonie fixe, mobile et de l'Internet de 1999 à 2007, Cameroun, 2007.

[10] Jason Cole, Helen Foster. Using Moodle, teaching with the popular open source course management system. O'REILLY, 2nd Edition, 2008.

[11] Xavier Pétard-Francis Forbeau. L'évolution des services numériques à partir de dispositifs nomades, ENS ULM, Esupday 9, 05 Février 2010.

\section{AUTHORS}

B. Batchakui, is a post graduate Computer Science Engineer and PhD student at ALOCO-LIRIMA at the National Advanced School of Engineering (ENSP) of the University of Yaoundé 1, PO Box 8390 YaoundéCameroon. He is now a Lecturer at the same Engineering School, teaching object-oriented programming, unified modeling language, software engineering and expert system. His research areas are e-learning Grid and Multiagents Systems (e-mail: bbatchakui@gmai.com).

T. N. Djotio PhD. is Doctor /Engineer in computer engineering and Lecturer at the National Advanced School of Engineering (ENSP) of the University of Yaoundé 1, PO Box 8390 Yaoundé-Cameroon. He is the Scientific Team Leader of MASECNES research team of LIRIMA. He is teaching information and network security, operating system, network technology and administration and system programming. His research areas are intelligent systems for network administration and security, threats investigation and mitigation, network and telecommunication protocol definition. (e-mail:tdjotio@gmail.com).

C. Tangha, is currently the head of the Department of the Computer Engineering at the National Advanced School of Engineering (ENSP) of the University of Yaoundé 1, PO Box 8390 Yaoundé-Cameroon. He is the Scientific Team Leader of ALOCO research team of LIRIMA. He has been teaching since 1976 at the ENSP. His research areas are software engineering, e-learning and artificial intelligence including expert systems, multiagent systems, and formal systems. He has directed many research projects, contributed to many conferences and seminars nationwide and at the international level. (email:ctangha@gmail.com).

This work was supported in part by LIRIMA-UY1/Cameroon.

This article is an extended version of a paper presented at the IEEE EDUCON20211 Conference, held from April 4th-6th, 2011, in Amman, Jordan. Received, April $28^{\text {th }}$, 2011. Published as resubmitted by the authors May $2^{\text {nd }}, 2011$. 